\title{
Enhanced structure of a double-pass erbium-doped fiber amplifier for multiple wavelength amplifications.
}

\begin{abstract}
We demonstrate an enhanced double-pass erbium-doped fiber amplifier for multichannel amplification. The multichannel selection is formed by combining a demultiplexer and a multiplexer together in the fiber-loop mirror. The structure is able to filter out the amplified spontaneous emission that saturates the amplifier gain in the small signal regime. The maximum average gain of $47.2 \mathrm{~dB}$ is obtained with a gain enhancement of $12.8 \mathrm{~dB}$ at a -50 $\mathrm{dBm}$ signal power per channel. The noise figure penalty is almost negligible for a signal power per channel of less than $-15 \mathrm{dBm}$.
\end{abstract}

Keyword: Amplification; Amplifiers (electronic); Erbium; Fiber lasers; Fiber optics; Optical waveguides. 